\title{
Transcriptional regulation of metastatic [Id]entity by KLF17
}

\author{
Marcin P Iwanicki and Joan S Brugge
}

Address: Department of Cell Biology, Harvard Medical School, 240 Longwood Avenue, Boston, MA 02115, USA.

Correspondence: Joan S Brugge. Email: Joan_Brugge@hms.harvard.edu

\begin{abstract}
A novel in vivo screening approach has identified $K L F 17$ as a key metastasis suppressor gene that acts through regulation of Id1 transcription factor-dependent induction of the epithelial-tomesenchymal transition.
\end{abstract}

The development and growth of a malignant tumor relies on molecular changes that affect the function of cellular proto-oncogenes and tumor suppressors [1]. Certain alterations are required constitutively during tumor initiation and progression, whereas others are more specifically associated with aspects of tumor metastasis - the spread of malignant cells to secondary tissues. Metastasis suppressors are proteins that specifically repress events associated with metastasis without affecting primary tumor growth [2]. In a recent paper in Nature Cell Biology, Huang and colleagues (Gumireddy et al. [3]) describe experiments that identify a new metastasis suppressor, KLF17.

\section{The course of metastasis}

Progression towards metastatic disease involves a distinct sequence of events. Tumor cells attract endothelial cells to promote angiogenesis; dissociate from the primary tumor mass and migrate towards endothelial cells; intravasate through endothelial cells and the surrounding matrix; enter the intra-tumoral vasculature; exit the vasculature at the secondary sites; infiltrate the new environment; and establish growing malignant colonies within a new organ [4]. These events are likely to be driven in large part by changes in transcriptional programs that affect expression of genes required for these processes (for example, genes that promote angiogenesis, cell adhesion and matrix proteolysis). These transcriptional changes are mediated by alterations in the tumor microenvironment [5] as well as by genetic alterations in proto-oncogenes and tumor suppressors [6]. For example, many reports have shown that oncogenic mutations in $\mathrm{H}-$ Ras or loss of $\mathrm{p} 53$ tumor suppressor function induce transcriptional programs that promote metastasis through alterations in the expression of genes required for the invasion of cancer cells into the surrounding tissues [4,710]. Transcription factors that promote the loss of epithelial characteristics, such as (cell-to-cell) adhesion, have also been proposed to play a key role in the initiation of metastasis.
The epithelial-to-mesenchymal transition (EMT) was initially described as a fundamental process that drives morphogenetic tissue movements in animal embryos [11]. Increasing evidence supports the hypothesis that epithelial tumors adopt EMT-like characteristics during the invasion of proximal tissues $[12,13]$. EMT involves the loss of apicobasal epithelial cell polarity and the development of a migratory polarity (leading edge and trailing edge) that promotes migration [14].The molecular hallmarks of EMT include the increased expression of transcription factors that contribute to the loss of cell adhesion through the downregulation of the cell adhesion molecule E-cadherin and increased proteolysis of the surrounding extracellular matrix as a result of the elevated expression of matrix metalloproteinases [14]. Cells that have undergone EMT in culture show an increased capability for invasive behavior in vitro and in vivo [12].

\section{Genome-wide screen for metastasis suppressors}

Gumireddy et al. [3] used a genome-wide screen to identify genes that induce metastasis when their expression is attenuated. Non-invasive mouse mammary tumor cells were transduced with a genome-wide short hairpin RNA (shRNA) library and implanted into mouse mammary fat pads. Lung metastases that developed in these animals were then analyzed by PCR for the most abundant shRNA transcripts, one of which was found to target expression of Krüppel-like transcriptional factor 17 (KLF17). Gumireddy et al. [3] then found that shRNA-mediated attenuation of KLF17 expression in the MCF-7 line of human nonmetastatic breast cancer cells promoted lung metastases in mice into which the treated cells were introduced. In addition, the overexpression of KLF17 in highly metastatic 4 $\mathrm{T} 1$ mouse breast cancer epithelial cells decreased the number of lung metastases in mice compared to control cells. These experiments provide strong evidence that $K L F 17$ is a new metastasis suppressor gene.

KLF17 belongs to the Sp (small protein)/KLF zinc-finger protein family. Other members of this family have been shown to regulate cell invasion in vitro [15]. For example, ectopic expression of $\mathrm{KLF}_{4}$ and/or $\mathrm{KLF}_{5}$ blocked the invasive behavior of esophageal cancer cells in vitro [15]. 


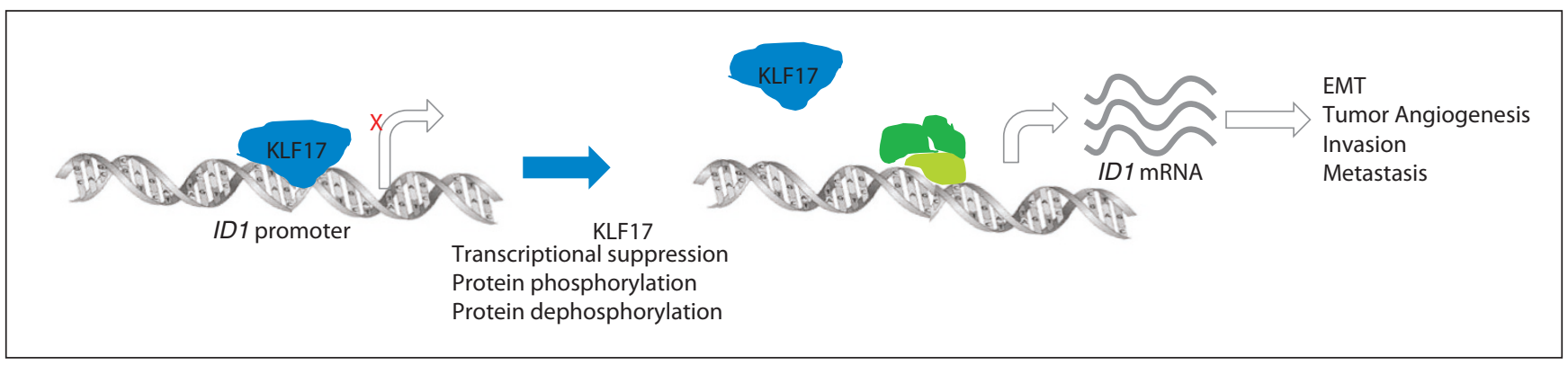

Figure 1

Regulation of ID1 expression by KLF17. ID1 transcription is suppressed by KLF17 binding to the ID1 promoter. Loss of expression of KLF17 by transcriptional suppression or inactivation by post-translational modification (possibly by phosphorylation or dephosphorylation) leads to transcription of ID1 through positive transcriptional regulators (shown in green) and induction of the multiple processes regulated by its protein product, Id1.

However, the exact mechanisms by which these proteins achieve their suppressive effect was not understood. Gumireddy et al. [3] employed microarray analysis of mRNAs in cells subjected to loss or gain of $K L F 17$ expression to provide insights into the mechanisms by which KLF family members suppress cell invasiveness and metastasis. They show in particular that shRNA-mediated attenuation of KLF17 levels in non-metastatic cells induces an EMT 'signature' of gene expression and increased expression of inhibitor of differentiation-1 (Id1), which is known to be a negative regulator of basic helix-loop-helix family transcription factors and to be involved in promoting EMT. Conversely, overexpression of KLF17 suppressed Id1 expression. The authors further show that KLF17 suppresses Id1 expression directly by binding to its promoter region. Id 1 and KLF17 have opposite effects on a tumor's ability to metastasize. Primary tumor samples with high Id1 and low KLF17 expression were highly metastatic, whereas those with higher expression of KLF17 and lower levels of Id1 were less metastatic. Taken together, these data support the conclusion that Id 1 plays a critical role in the regulation of metastasis by KFL17.

\section{Id1 and human cancer}

$I D 1$ is overexpressed in various human cancers, including endometrial, ovarian, prostate and breast cancers [16], and had previously been shown to promote invasion and metastasis [10]. In tubular epithelial cells, $I D 1$ was shown to be required for transforming growth factor- $\beta$-mediated loss of E-cadherin expression (a molecular hallmark of EMT) [17]. ID1-mediated downregulation of E-cadherin occurs through the direct binding of Id1 protein to the transcription factor HEB (HELA E-box binding factor), which prevents HEB from accessing the E-cadherin promoter [17]. Interestingly, Gumireddy et al. [3] show that the loss of KLF17 function in the NMuMg mouse mammary epithelial cell line transformed by the oncogene V12H-Ras enhances its capacity to produce lung metastases and drives metastasis to several other organs. Given that V12H-Ras induces EMT [18-20], these results suggest that processes in addition to EMT contribute to the promotion of metastasis by Id1. For example, Id1-induced metastasis could also involve tumor vascularization, as Id 1 promotes production of vascular endothelial growth factor (VEGF) in prostate cancer cells [21]. Taken together, these studies suggest a model in which loss of KFL17 leads to induction of $I D 1$, which could promote primary tumor vascularization via VEGF production and initiate invasion through EMT-associated processes, including loss of E-cadherin adhesions and increased activation of matrix metalloproteinases.

One important question raised by the work of Gumireddy et al. [3] is whether the regulation of KLF17 is entirely transcriptional or if its activity is also regulated posttranslationally, and what upstream factors control gene expression or protein activity. The transcriptional activity of $\mathrm{Sp} 1 / \mathrm{KLF}$ family members has been shown to be regulated post-translationally by phosphorylation $[8,22]$. A search for phosphorylation-site motifs using Scansite [23] predicts a high-stringency target site for phosphorylation by protein kinase $\mathrm{C}-\varepsilon(\mathrm{PKC} \varepsilon)$ within the amino-terminal region of KLF17. Given that the activity of some Sp1/KLF family members is known to be regulated by PKC isoforms $[8,24-26]$, it is plausible that the ability of KLF17 to suppress ID1 transcription could be either positively or negatively tuned by some PKCs (Figure 1). However, future experiments addressing the mechanisms of KLF17 regulation will be needed to fill this gap in our understanding of metastatic progression.

The findings of Gumireddy et al. [3] highlight the feasibility and the value of in vivo loss-of-function screens. As the authors point out, these screens should be carried out in models other than breast cancer, as distinct transcription factors might play a role in the regulation of invasion and metastasis in different cancer types. 


\section{References}

1. Fidler IJ: The pathogenesis of cancer metastasis: the 'seed and soil' hypothesis revisited. Nat Rev Cancer 2003, 3:453458.

2. Smith SC, Theodorescu D: Learning therapeutic lessons from metastasis suppressor proteins. Nat Rev Cancer 2009, 9:253-264.

3. Gumireddy K, Li A, Gimotty PA, Klein-Szanto AJ, Showe LC, Katsaros D, Coukos G, Zhang L, Huang Q: KLF17 is a negative regulator of epithelial-mesenchymal transition and metastasis in breast cancer. Nat Cell Biol 2009, 11:12971304

4. Nguyen DX, Bos PD, Massague J: Metastasis: from dissemination to organ-specific colonization. Nat Rev Cancer 2009, 9:274-284

5. Kizaka-Kondoh S, Tanaka S, Harada H, Hiraoka M: The HIF-1active microenvironment: an environmental target for cancer therapy. Adv Drug Deliv Rev 2009, 61:623-632.

6. Weinberg RA: Mechanisms of malignant progression. Carcinogenesis 2008, 29:1092-1095.

7. Rak J, Mitsuhashi Y, Bayko L, Filmus J, Shirasawa S, Sasazuki T, Kerbel RS: Mutant ras oncogenes upregulate VEGF/VPF expression: implications for induction and inhibition of tumor angiogenesis. Cancer Res 1995, 55:4575-4580.

8. Pal S, Datta K, Khosravi-Far R, Mukhopadhyay D: Role of protein kinase Czeta in Ras-mediated transcriptional activation of vascular permeability factor/vascular endothelial growth factor expression. J Biol Chem 2001, 276:2395-2403.

9. Wang SP, Wang WL, Chang YL, Wu CT, Chao YC, Kao SH, Yuan A, Lin CW, Yang SC, Chan WK, Li KC, Hong TM, Yang PC: p53 controls cancer cell invasion by inducing the MDM2-mediated degradation of Slug. Nat Cell Biol 2009, 11: 694-704.

10. Fong $S$, Itahana $Y$, Sumida $T$, Singh J, Coppe JP, Liu $Y$, Richards PC, Bennington JL, Lee NM, Debs RJ, Desprez PY: Id-1 as a molecular target in therapy for breast cancer cell invasion and metastasis. Proc Natl Acad Sci USA 2003, 100: 13543-13548.

11. Thiery JP: Epithelial-mesenchymal transitions in development and pathologies. Curr Opin Cell Biol 2003, 15:740-746.

12. Polyak K, Weinberg RA: Transitions between epithelial and mesenchymal states: acquisition of malignant and stem cell traits. Nat Rev Cancer 2009, 9:265-273.

13. Mani SA, Guo W, Liao MJ, Eaton EN, Ayyanan A, Zhou AY, Brooks M, Reinhard F, Zhang CC, Shipitsin M, Campbell LL, Polyak K, Brisken C, Yang J, Weinberg RA: The epithelialmesenchymal transition generates cells with properties of stem cells. Cell 2008, 133:704-715.
14. Friedl $P$, Wolf $K$ : Tumour-cell invasion and migration: diversity and escape mechanisms. Nat Rev Cancer 2003, 3:362374 .

15. Yang Y, Goldstein BG, Chao HH, Katz JP: KLF4 and KLF5 regulate proliferation, apoptosis and invasion in esophageal cancer cells. Cancer Biol Ther 2005, 4:1216-1221.

16. Perk J, lavarone A, Benezra R: Id family of helix-loop-helix proteins in cancer. Nat Rev Cancer 2005, 5:603-614.

17. Li Y, Yang J, Luo JH, Dedhar S, Liu Y: Tubular epithelial cell dedifferentiation is driven by the helix-loop-helix transcriptional inhibitor ID1. J Am Soc Nephrol 2007, 18:449-460.

18. Edme N, Downward J, Thiery JP, Boyer B: Ras induces NBT-I epithelial cell scattering through the coordinate activities of Rac and MAPK pathways. J Cell Sci 2002, 115:2591-2601.

19. Boyer B, Roche S, Denoyelle M, Thiery JP: Src and Ras are involved in separate pathways in epithelial cell scattering. EMBO J 1997, 16:5904-5913.

20. Larue L, Bellacosa A: Epithelial-mesenchymal transition in development and cancer: role of phosphatidylinositol 3' kinase/AKT pathways. Oncogene 2005, 24:7443-7454

21. Ling MT, Lau TC, Zhou C, Chua CW, Kwok WK, Wang Q, Wang $X$, Wong YC: Overexpression of Id-1 in prostate cancer cells promotes angiogenesis through the activation of vascular endothelial growth factor (VEGF). Carcinogenesis 2005, 26:1668-1676.

22. Tan NY, Khachigian LM: Sp1 phosphorylation and its regulation of gene transcription. Mol Cell Biol 2009, 29:2483-2488.

23. Scansite [http://scansite.mit.edu/]

24. Black AR, Black JD, Azizkhan-Clifford J: Sp1 and kruppel-like factor family of transcription factors in cell growth regulation and cancer. J Cell Physiol 2001, 188:143-160.

25. Kavurma MM, Santiago FS, Bonfoco E, Khachigian LM: Sp1 phosphorylation regulates apoptosis via extracellular FasL-Fas engagement. J Biol Chem 2001, 276:4964-4971.

26. Tan NY, Midgley VC, Kavurma MM, Santiago FS, Luo X, Peden R, Fahmy RG, Berndt MC, Molloy MP, Khachigian LM: Angiotensin II-inducible platelet-derived growth factor-D transcription requires specific Ser/Thr residues in the second zinc finger region of Sp1. Circ Res 2008, 102:e3851.

Published: 30 November 2009

doi:10.1186/gb-2009-10-11-244

(C) 2009 BioMed Central Ltd 